\title{
Measurement of XeI and XeII velocity in the near exit plane of a low-power Hall effect thruster by light induced fluorescence spectroscopy
}

\author{
Y.Dancheva, \\ CNISM, University of Siena, CSC and DSFTA, via Roma 56, 53100 Siena, Italy \\ V.Biancalana \\ CNISM, University of Siena, CSC and DIISM, via Roma 56, 53100 Siena, Italy \\ D.Pagano, F.Scortecci \\ Aerospazio Tecnologie Srl., via Provinciale Nord 42a, 53040 Rapolano Terme (SI), Italy
}

\begin{abstract}
Near exit plane non-resonant light induced fluorescence spectroscopy is performed in a Hall effect low-power Xenon thruster at discharge voltage of $250 \mathrm{~V}$ and anode flow rate of $0.7 \mathrm{mg} / \mathrm{sec}$. Measurement of the axial and radial velocity components are performed, exciting the $6 s^{2}[3 / 2]_{2}^{o} \rightarrow 6 p^{2}[3 / 2]_{2}$ transition at $823.16 \mathrm{~nm}$ in XeI and the $5 d[4]_{7 / 2} \rightarrow 6 p[3]_{5 / 2}^{\circ}$ transition at $834.724 \mathrm{~nm}$ in XeII. No significant deviation from the thermal velocity is observed for XeI. Two most probable ion velocities are registered at a given position with respect to the thruster axis, which are mainly attributed to different areas of creation of ions inside the acceleration channel. The spatial resolution of the setup is limited by the laser beam size (radius of the order of $0.5 \mathrm{~mm}$ ) and the fluorescence collection optics, which have a view spot diameter of $8 \mathrm{~mm}$.

PACS numbers: 42.62.Fi; 42.79.Qx; 52.70.-m
\end{abstract}

\section{INTRODUCTION}

Light induced fluorescence (LIF) spectroscopy is a powerful diagnostic technique in thruster characterization. Velocity measurements based on the Doppler shift of a given species in the plume require accurate and finely calibrated laser-based set-ups. Nevertheless, there are unquestionable advantages that make this technique very useful. Even though the experimental set-ups are rather complex and delicate, the advanced specifications and the robustness of currently available diode laser sources in terms of spectral linewidth, frequency and intensity noises, power, and possibility of stable and reliable optical fiber coupling make it possible to engineer robust and user-friendly devices. It is worth pointing out the unparalleled properties of the LIF velocimetry, which is very unintrusive, has high spatial resolution, offers the possibility to extract information even where the plasma itself is created, is species selective, and permits the simultaneous detection of multiple velocity components. Excellent results have been obtained with LIF-based techniques in various types of electric thrusters, with reference to the $6 \mathrm{~kW}$ power range [1, 2], the $1 \mathrm{~kW}$ range 3,4$]$ and low to medium power range [5 7 ].

The aim of this paper is to characterize a low-power Hall thruster by investigating the behaviour of the near exit plane velocity components, using LIF spectroscopy in XeI and XeII. For this purpose a multiple channel LIF set-up was built using different free running diode lasers. Details of the laser system, the detection/illumination optics, and the spectral calibration system are provided. A spectral analysis and the determination of the velocity distribution are presented and discussed.

\section{APPARATUS}

The measurements presented here are performed in a non-magnetic stainless steel vacuum chamber of $1.3 \mathrm{~m}$ in diameter and $3 \mathrm{~m}$ in length. The vacuum pumping is provided by a completely oil free system. In particular, during tests the pumping is performed by a cryogenic system which includes a commercial cryopump and a customsized cryopanel cooled to below $50 \mathrm{~K}$ by a cryorefrigerator, giving a residual pressure of around $4 \times 10^{-5} \mathrm{mbar}$ (measured with an ionization gauge calibrated for Nitrogen and corrected for Xe using the gas correction factor recommended by the gauge manufacturer).

The thruster under investigation is $100 \div 200 \mathrm{~W}$ class Hall Effect Thruster (HET). The external diameter of the acceleration chamber is $40 \mathrm{~mm}$, while the internal diameter is $28 \mathrm{~mm}$. The cathode is mounted so that the angle between the thruster output plane and the cathode axis is $45^{\circ}$. The cathode outlet orifice is located approximately $35 \mathrm{~mm}$ radially and $20 \mathrm{~mm}$ downstream from the center of the channel exit. The thruster is mounted on two electrically driven, micrometric linear stages in order to allow it to move in two directions (vertical and horizontal).

\section{A. Diode laser source}

Non-resonant LIF spectroscopy is performed in XeI and in XeII exciting and detecting the transitions denoted in Fig 1 .

For this purpose a diode laser system was built using two standard Fabry-Perot index-guided semiconductor chips: (i) laser diode chip (LD1), delivering up to 

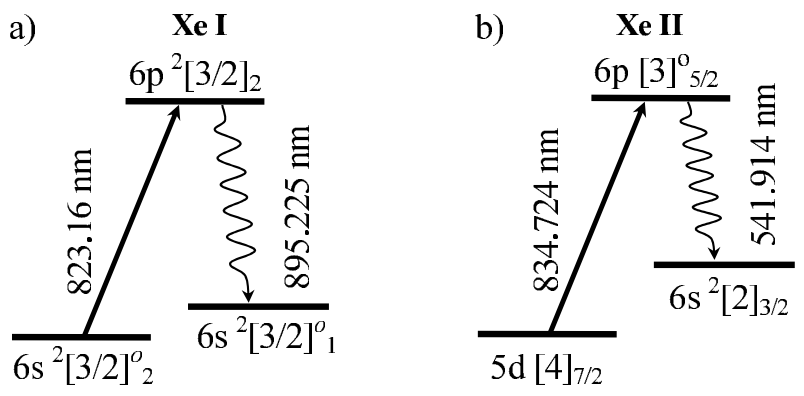

FIG. 1: Energy diagrams of the transitions used for nonresonant LIF spectroscopy in a) XeI and b) XeII. The wavelengths are given in air.

$150 \mathrm{~mW}$ of optical power and emitting around $834 \mathrm{~nm}$, and (ii) LD2, delivering $30 \mathrm{~mW}$ and emitting around $823 \mathrm{~nm}$. The diode laser chips are placed in a commercial diode laser head and their output beams are launched into a single-mode optical fiber (see Fig[2). The head allows PID thermostatation with resolution of $0.01^{\circ} \mathrm{C}$. The laser beam size is matched to the single mode optical fiber by means of a two-lens optical system, followed by a commercial launcher in order to maximize the power coupling. The laser power is intensity-modulated in the range of $200 \mathrm{~Hz}$ using a mechanical chopper. The fiber coupling efficiency achieved is of the order of $50 \%$ and the laser power is subsequently divided into 4 outputs using a 1x4 single mode fiber splitter. One of the outputs is used to perform spectroscopy in a galvatron spectroscopic lamp aimed at providing a calibrated-wavelength references. The other three fibers are available for LIF spectroscopy of the HET positioned inside the vacuum chamber. Details of the coupling to the vacuum chamber and of the illumination system used inside the chamber are reported in Sec $\llbracket$.

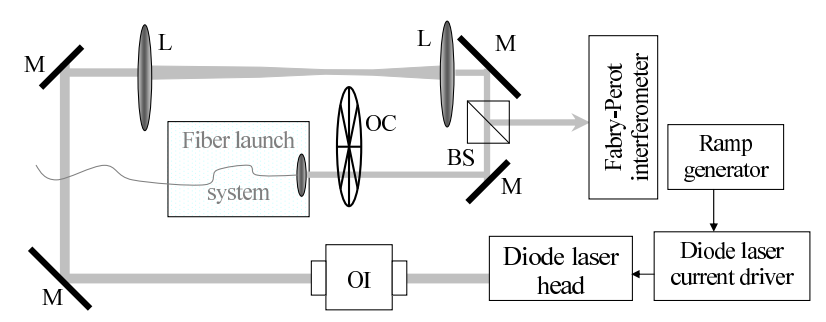

FIG. 2: Schematic of the diode laser source: OI-optical isolator; M-mirror; L-lens; OC-optical chopper; BS-beam splitter.

Both laser diodes deliver continuous, mode-hop-free tuning in ranges broad enough to perform simultaneous spectroscopy in the stationary reference and in the plume of the thruster. Specifically, the LD1 laser can be continuously tuned over more than $40 \mathrm{GHz}$ and the LD2 over more than $15 \mathrm{GHz}$ in the free running regime.

\section{B. Reference signals}

The atomic/ionic velocities are determined on the basis of the Doppler shift of a given known line, measured with respect to a suitable reference line. The choice of the reference line must be made considering the frequency separation between it and the expected position of the shifted fluorescence line, as this determines the continuous (mode-hop-free) frequency scan of the laser diode to be performed. Thus the position of the reference line, together with the mode-hop-free detuning of the laser, limits the range of measurable shifts and consequently the measurable particle velocity.

In the case of atomic velocity measurement, the reference line is easily chosen, being the same as the unshifted fluorescence line of a stationary discharge, since the velocity of the neutral is essentially thermal. In the case of ion velocimetry, and in the absence of an ionic stationary reference line, a well-known-wavelength atomic line can be used for reference. In some works (see, for example, [5, 8] on LIF velocimetry exciting the ionic transition at $834.724 \mathrm{~nm}$ the Doppler shift is determined with respect to the atomic line at $834.68217 \mathrm{~nm}$, corresponding to the transition $6 s^{\prime}[1 / 2]_{1}^{o} \rightarrow 6 p^{\prime}\lfloor 3 / 2]_{2}$ [5, 9]. Consequently diode frequency scans of the order of $40 \mathrm{GHz}$ must be performed without laser mode-hops. Manzella and colleagues [10] use the laser back reflection onto the thruster itself as a reference line, which further increases the mode-hop-free tuning range required. The unshifted ion line in a stationary discharge is used as well as a reference line, thus reducing the mode-hop-free range by a factor of 2 (see, for example, [3]). In this work a different (more advantageous) atomic reference line is proposed (see details at the end of this section).

The experimental set-up for LIF reference signal detection used in this work is presented in Fig, 3, As shown, the optogalvanic (OG) signal is recorded as a slight variation in the voltage drop registered at the ballast resistor $R_{b}$. The use of this kind of signal facilitates the task of transduction by making the atomic response signal immediately available as an electric one. With a supply voltage of $120 \mathrm{~V}$ (around half of it across the discharge lamp) and $R_{b}=600 \mathrm{k} \Omega$ the galvatron current is of about $0.1 \mathrm{~mA}$ and the optogalvanic signal is of the order of $30 m V_{p-p}$ at $834.68217 \mathrm{~nm}$.

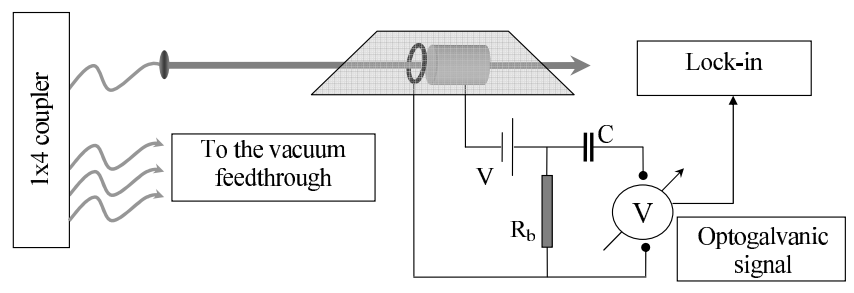

FIG. 3: Reference signal arrangement. $\mathrm{C}=470 \mathrm{nF}$; $\mathrm{Rb}=600 \mathrm{k} \Omega ; \mathrm{V}=120 \mathrm{~V} ; \mathrm{I}=0.1 \mathrm{~mA}$. Lock-in is referenced to the optical chopper frequency. 
The phase-sensitive detection (PSD) technique is employed for low-noise demodulation of the optogalvanic signal using a lock-in amplifier, with a typical integration time of the order of $1 s \div 3 \mathrm{~s}(6 \mathrm{~dB} /$ oct $\div 12 \mathrm{~dB} /$ oct output filter). As discussed below, such a long integration time is chosen due to the need to average out the thruster's noise, rather than the optogalvanic lamp discharge noise.

Continuous frequency tuning is performed using a triangular wave generator applied to the modulation input of the diode laser current driver. A maximum $1 V_{p-p}$ triangular wave signal is applied to the diode laser current driver input, causing a driving current variation of $20 \mathrm{~mA}$. Its frequency is very low (of the order of few $\mathrm{mHz}$ ) so that the junction current is varied slowly with respect to the measuring time. According to the laser-driver modulation conversion factor (input modulation voltage to junction current) and to the laser diode chip response (junction current to optical frequency), the scan produces a continuous sweep of the optical frequency. This sweep is accurately monitored with the help of a confocal FabryPerot (FP) interferometer with a free spectral range of $1.47 \mathrm{GHz}$. The signals from the FP cavity and from the reference lamp are acquired simultaneously, with the LIF signal being demodulated by a second lock-in amplifier, so that they provide a precise relative (the FP) and absolute (the stationary discharge) calibration of the frequency scale.

Examples of the reference curves are shown in Fig,4 The laser power illuminating the discharge lamp is attenuated down to $2 \mathrm{~mW}$ using a neutral density filter. Fig 4
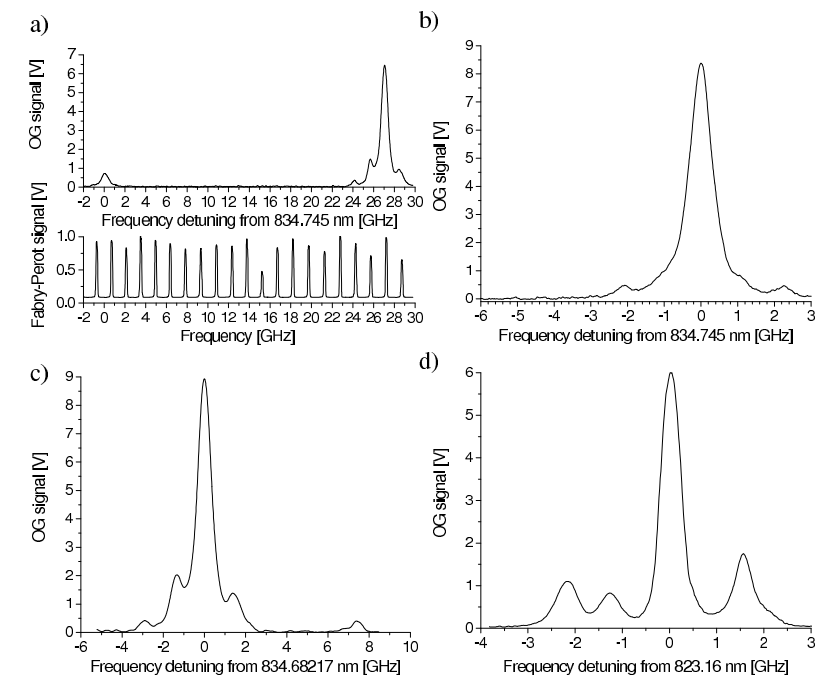

FIG. 4: Reference optogalvanic signals at: b) $834.745 \mathrm{~nm} \mathrm{c}$ ) $834.68217 \mathrm{~nm} \mathrm{~d}) 823.16 \mathrm{~nm}$ and a) at both $834.68217 \mathrm{~nm}$ and $834.745 \mathrm{~nm}$ together with the Fabry-Perot frequency comb.

shows the relevant lines available for the frequency scaleoffset calibration and includes (plot a) the Fabry-Perot comb of peaks used for frequency scale-factor calibration. Besides the reference line, at $823.16 \mathrm{~nm}$ for XeI velocimetry (plot d) and at $834.68217 \mathrm{~nm}$ for XeII velocimetry (plot c), the XeI has a weaker but clearly detectable line at $834.745 \mathrm{~nm}$ (transition $6 s^{2}[3 / 2]_{1} \rightarrow 8 s^{2}[3 / 2]_{1}^{o}$ ). This last line (plot b) is used as a reference line for XeII velocimetry in this work, as it is $9 \mathrm{GHz}$ red-shifted (the Doppler shift of the axial velocity of the moving ionic particles is also red) with respect to the stationary ionic one (at $834.724 \mathrm{~nm}$ ). The fact that this reference line is closer to the shifted ionic one decreases the necessary frequency tuning range by an other factor of 2 , thus speeding up the measurement time. The weaker amplitude is not problematic thanks to the good signal-to-noise ratio given by the OG signal.

\section{Light collection arrangement and data acquisition}

Two of the outputs of the $1 \times 4$ fiber splitter are coupled to illuminators placed inside the vacuum chamber using a four-port vacuum feedthrough. The optical power losses through the vacuum feedthroughs are significant for three out of four. Power loss levels in excess of $70 \%$ are measured for three feedthroughs and $30 \%$ for the other one. Inside the vacuum chamber, high-vacuum single mode fibers are used. The laser beam, applied in the axial $(\hat{z})$ direction, is collimated using an adjustable focal length collimator, providing a beam waist of $0.8 \mathrm{~mm}$ (diameter at $1 / e^{2}$ intensity) at a distance of $1 \mathrm{~m}$ (the distance between the fiber output and the thruster output plane). The fluorescence signal is collected using a multimode fiber equipped with collimation optics $(22 \mathrm{~mm}$ clear aperture (CA) collimator), which determines the lowest spatial selectivity (the highest view spot diameter). The view spot diameter is adjusted using a remotely controlled multiple-diameter diaphragm ranging from $18 \mathrm{~mm}$ to $8 \mathrm{~mm}$, placed in the proximity of the collimator entrance.

The collected light is extracted from the vacuum chamber through a multi mode fiber vacuum feedthrough and is directed to a $0.5 \mathrm{~m}$ monochromator. The monochromator slits are adjusted to set maximum transmittance at $895.22 \mathrm{~nm}$ for LIF in XeI, and at $541.914 \mathrm{~nm}$ for LIF in XeII, with a spectral width of about $1.5 \mathrm{~nm}$, in order to perform non-resonant LIF spectroscopy and improve the signal-to-noise ratio by rejecting broad spectrum radiation. This spectral width abundantly exceeds that of the diode laser and the LIF signal, so that the monochromator acts as an adjustable band-pass filter with a flatresponse in the range of interest and has no effect on the spectral features measured. A high gain, ultra-low noise photomultiplier tube (PMT) is used as a detector at the monochromator output.

Four signals are acquired simultaneously: the triangular wave signal applied to the diode laser current driver; the Fabry-Perot fringes; the optogalvanic signal, and the LIF signal, both at the output of the lock-in amplifiers. The ramp signal is used to determine the direction of the frequency ramp. The Fabry-Perot signal is used to lin- 
earize the diode laser frequency scan and to establish a calibrated relative frequency scale. The optogalvanic signal is used to determine the frequency shift with respect to the atomic line nearest to the ionic one and thus to calibrate the frequency scale offset. Multiple measurements are performed at each $\hat{y}, \hat{z}$ position. This helps to improve the signal-to-noise ratio by averaging and provides uncertainty estimation in terms of the sample standard deviation of the inferred velocity.

\section{RESULTS}

\section{A. LIF velocimetry in $\mathrm{XeI}$}

The performance, lifetime and operational stability of a HET depend on its neutral propellant flow parameters. For example, the azimuthal uniformity of the number density at the propellant distributor exit plane has been studied in detail [11 13] as an important parameter to obtain efficient performance of the thruster.

In this work the axial velocity component of Xe atoms is only recorded for the purpose of illustrating the setup's potential for multiple species interrogation. The thruster channel is illuminated at $823 \mathrm{~nm}$ (laser power of $1.6 \mathrm{~mW}$ ) and the fluorescence is collected at a distance of $z=-20 \mathrm{~mm}$ from the thruster output plane, with a collimator view point diameter of $18 \mathrm{~mm}$. The LIF signal at $895 \mathrm{~nm}$ is presented in Fig 5 (for a Xe anode flow of $0.8 \mathrm{mg} / \mathrm{sec}$ ). The spectrum of the signal is determined by the multiple Xe isotopes and the hyperfine structure (see for comparison Fig 4d). The single axial velocity component was found to be essentially thermal. Similar results have been obtained for $\mathrm{Xe}$ anode flows down to $0.6 \mathrm{mg} / \mathrm{sec}$. The small negative axial component measured, of about $30 \mathrm{~m} / \mathrm{sec}$, was attributed to the anode temperature increase and/or collisions with Xe ions.

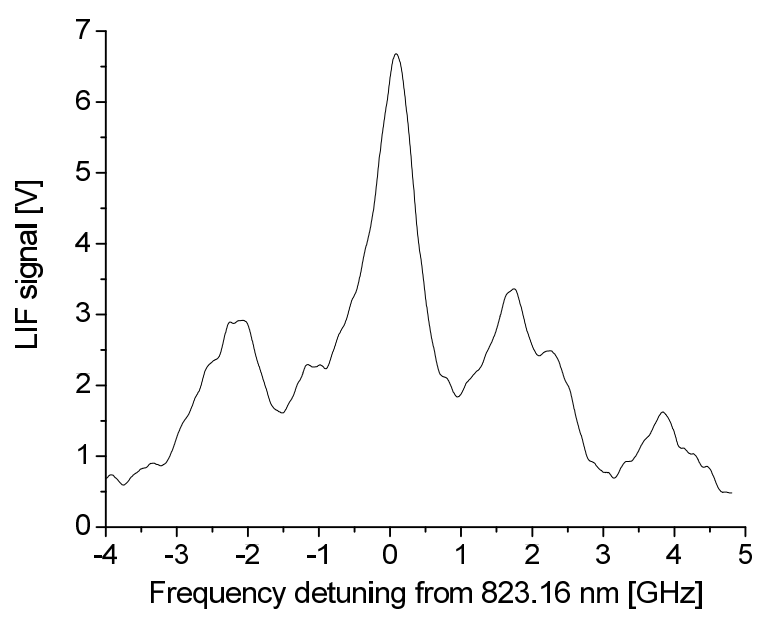

FIG. 5: LIF signal at $895 \mathrm{~nm}$ for the HET at: V=250 V; $\mathrm{I}=0.8 \mathrm{~A}$; Xe anode flow $0.8 \mathrm{mg} / \mathrm{sec}$; lock-in $\tau=3 \mathrm{sec}$; (output filter at $12 \mathrm{~dB} /$ oct); laser power of $1.6 \mathrm{~mW}$ in axial direction.

\begin{tabular}{||l|l||}
\hline Anode mass flow rate & $0.7 \mathrm{mg} / \mathrm{sec}$ \\
\hline Cathode mass flow rate & $0.2 \mathrm{mg} / \mathrm{sec}$ \\
\hline Anode potential & $250 \mathrm{~V}$ \\
\hline Anode current & $0.67 \mathrm{~A}$ \\
\hline
\end{tabular}

TABLE I: Nominal thruster operating conditions

\section{B. LIF velocimetry in $\mathrm{XeII}$}

Two velocity component measurements were carried out in order to determine ion velocity along the two orthogonal directions (axial and transverse directions). One laser beam, with a power of $8.5 \mathrm{~mW}$ and diameter of $0.8 \mathrm{~mm}$ (at the thruster plane), is used to determine the velocity component along the direction perpendicular to the thruster exit plane. A second laser beam, slightly larger in diameter $(1 \mathrm{~mm})$ and lower in power $(3 \mathrm{~mW})$, travels along the transverse direction, providing the radial component of the velocity if the beam lies on one of the symmetry planes of the thruster (approximated with a cylinder if the presence of the cathode is neglected geometrically). The schematic of the laser illumination system set-up inside the vacuum chamber is sketched in Fig,6, together with the location of the view spot with respect to the thruster output plane and the cathode position. The spontaneous and laser-induced fluorescence is collected with the multimode optical fiber (positioned at around $x=500 \mathrm{~mm}$ from the thruster) through the $8 \mathrm{~mm}$ diaphragm (see Fig 6). The intersection of the laser interaction volume with the view spot forms the light-plume detection volume, of less than $1 \times 1 \times 8 \mathrm{~mm}$.

Table 1 gives the nominal thruster operating conditions.

A two-axis translation system makes it possible to move the thruster in the $(\hat{x}, \hat{y})$ plane with respect to the fixed measurement optical system. The axial and transverse velocity components can be measured on all the points lying on this plane parallel to the thruster exit plane and $37 \mathrm{~mm}$ from it, i.e. $20 \mathrm{~mm}$ beyond the cathode position.

Both laser beams (applied in axial and radial directions) are aligned so as to cross at the center of the view spot. Due to the noise coming from the thruster itself, a long time constant must be used for LIF phase sensitive detection and thus no strict requirements are imposed for the diode laser intensity modulation frequency (using the mechanical chopper). As mentioned in Sec IIB. the diode laser optical frequency is scanned slowly using a triangular wave generator. LIF signals such as those presented in Fig [7 can be registered using a lock-in amplifier (we use a Stanford SR830 lock-in amplifier) with a time constant of $1 \mathrm{sec}$ and a $12 \mathrm{~dB} /$ oct output filter. The integration time set in conjunction with the diode laser frequency sweep width makes it necessary to use triangular wave signals with a frequency of the order of $2 \mathrm{mHz}$ and thus a measurement time of the order of $4 \mathrm{~min}$ (one 


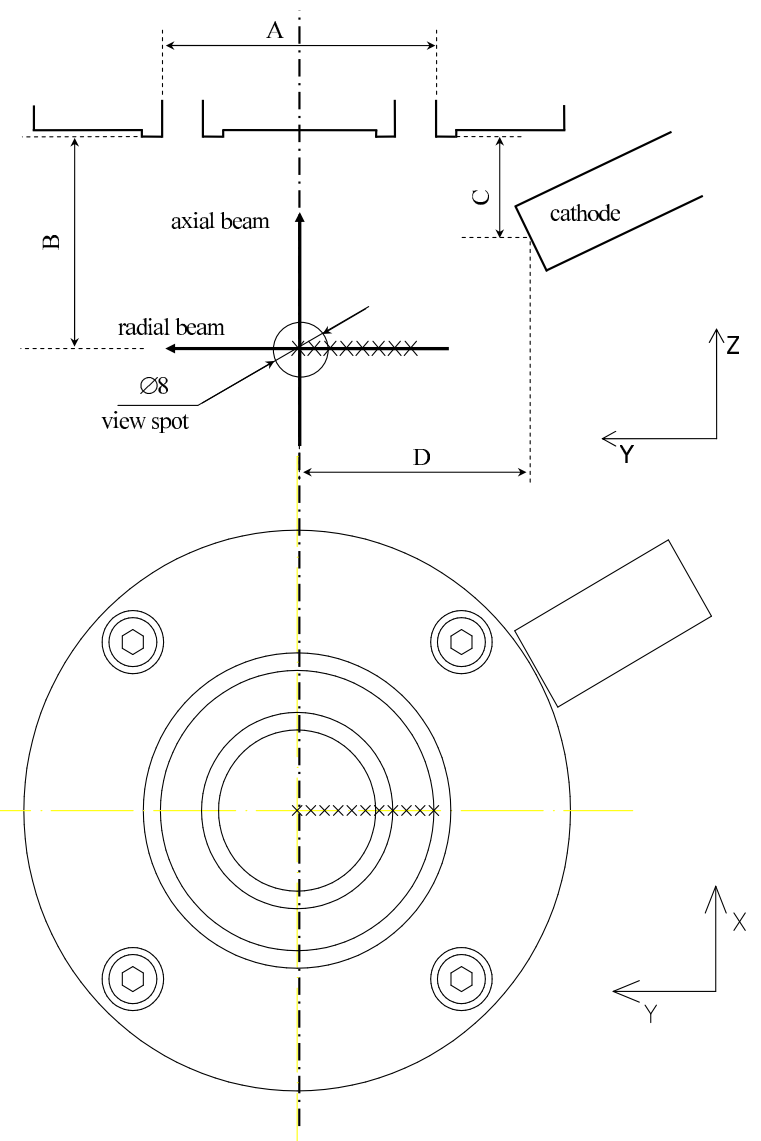

FIG. 6: Top and front view of the experimental arrangement inside the vacuum chamber $(A=40 \mathrm{~mm}, \mathrm{~B}=37 \mathrm{~mm}$, $\mathrm{C}=17 \mathrm{~mm}, \mathrm{D}=35 \mathrm{~mm}$ ). The position of the view spot is presented with respect to the thruster output plane. The various $y$ positions of the center of the thruster with respect to the illumination system are marked with $\times$. The origin of the co-ordinate system is positioned at the center the thruster.

scan).

With our experimental parameters the contrast of the LIF signal (determined as the ratio between the ac rms amplitude given by the lock-in and the PMT dc signal) is of the order of $1 \%$. The axial LIF signal presents a single, red-shifted peak at $18.5 \mathrm{GHz}$, corresponding to the most probable velocity of $15.4 \mathrm{~km} / \mathrm{sec}$ (calculated as $\vartheta=\Delta \nu / \lambda$ and corresponding to $\approx 163 \mathrm{eV}$ ) in the opposite to the laser beam direction and for $\mathrm{y}=0$. Two well-separated components of the radial velocity are observed at the center of the thruster, as well as at any displacement in the $\hat{y}$ direction (see Fig, 8 ). The radial nonzero velocity components show quite asymmetric peaks at $24 \mathrm{eV}$ (radial component in the direction of the cathode) and nearly $5 \mathrm{eV}$ (in the opposite direction with respect to the cathode).

The fluorescence spectrum changes in terms of both shape and peaks positions as it moves away from the center of the thruster exit plane, reflecting the decrease

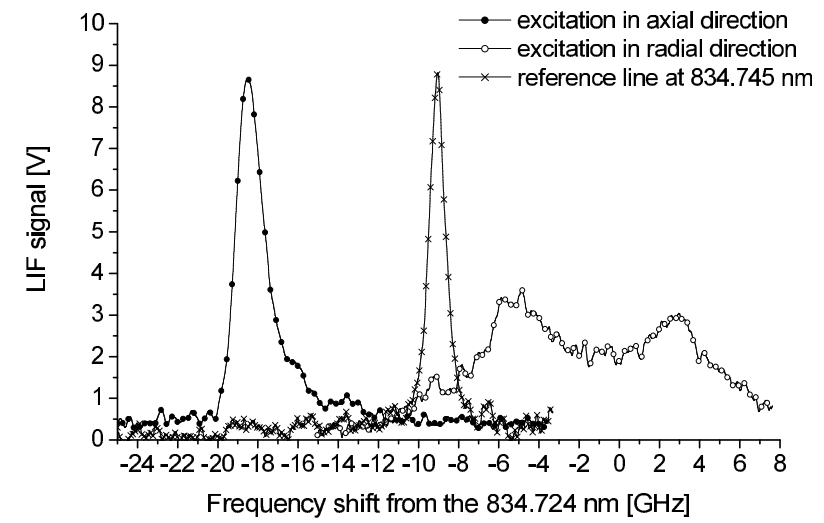

FIG. 7: Examples of the LIF signals $($ at $y=0)$ for laser excitation in both axial and radial directions, in scale.

of the ion density and the variation in the distribution of the ion velocity. The presence of double axial and radial velocity components is registered for $\mathrm{y} \geq-8 \mathrm{~mm}$. Double radial velocity components at $\mathrm{y}=0$ are likely due to overlapping of the ion flows coming from opposite sides of the accelerating channel. The presence of multiple velocity components is also found for the axial component. Fig 8 synthetically represents this behaviour, showing the most probable axial and radial velocity as a function of the $\hat{y}$ position. In particular, concerning the axial velocity, a single peak is present up to $\mathrm{y}=-4 \mathrm{~mm}$ from the center along the $\hat{y}$ direction the peak width increases progressively. At $\mathrm{y}=-6 \mathrm{~mm}$ a second peak appears, not yet well separated from the first one. Beyond this distance, a second peak becomes clearly visible.

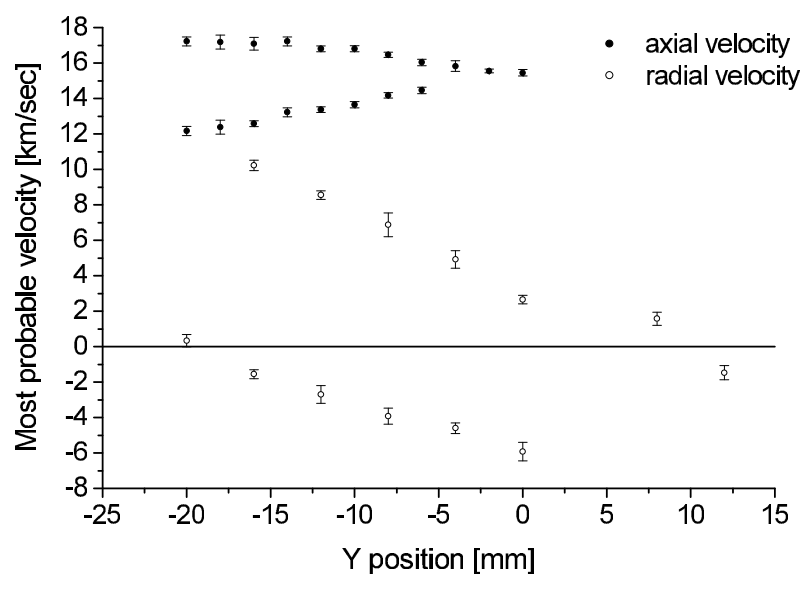

FIG. 8: Axial and radial components of the XeII velocity as a function of the $\hat{y}$ position. The error bars are the meansquare-root deviation over several measurements.

Concerning the axial velocity, a single velocity peak is observed for only $5 \mathrm{~mm}$ away from the center of the thruster. The broadening of the peak towards the lower velocities (corresponding to a decrease in the mean velocity) and subsequent appearance of two most probable velocities occur when the thruster in displaced in the $\hat{y}$ 
direction. It should be pointed out that the relative intensity of the two axial peaks is comparable.

The distribution behaviour of the radial velocity as a function of the radial displacement is different. There is a big discrepancy between the most probable velocity and the mean velocity. The mean radial velocity value as a function of the $\hat{y}$ displacement is close to zero. The radial LIF signal appears as a broad plateau with two side peaks of different intensities at distances bigger than $y=-4 \mathrm{~mm}$. When both are present, the intensity ratio between the two most probable radial velocities is of the order of 2 .

\section{CONCLUSIONS}

A set-up was built to measure multiple velocity components in XeI and XeII, based on Doppler shift measurements of LIF signals. The good spatial resolution permitted detailed investigation of the plume at centimetric distances from the exit plane of the thruster. Both XeI and XeII velocities were measured. No significant discrepancy from a thermal velocity was found in the case of XeI. In the case of XeII, and at a distance of $z=-$ $37 \mathrm{~mm}$, a map of the axial and radial velocity could be drawn and it was determined that XeII is accelerated to approximately $163 \mathrm{eV}$ at the center of the thruster. The poorer radial spatial selectivity with respect to the axial one made it difficult to establish whether the $24 \mathrm{eV}$ peak contributed to the maximum energy together with the axial peak.

A multiple channel LIF spectroscopy set-up was realized with the help of optical fibers. The system offers LIF spectroscopy (and thus accurate velocimetry) in both XeI and XeII by changing only the diode laser head. The possibility of using the transition at $834.745 \mathrm{~nm}$ as a reference was demonstrated, bringing the advantage of narrower diode laser frequency scanning, thus shortening the measuring time with respect to other reference lines and relaxing the requirements in terms of laser modehop-free tuning.

\section{ACKNOWLEDGMENTS}

The research was co-financed by Tuscany's Regional administration under the regional R\&D program, POR CreO FESR 2007-2013 Bando Unico R\&S 2008, whose support is gratefully acknowledged. The authors would like to acknowledge Dr. Giovanni Coduti for his help in building the experimental set-up, Mr. Simone Scaranzin for the tests carried out at Aerospazio Tecnologie Srl, and thank Emma Thorley for reviewing the manuscript. Y.D. and V.B. thank C.Stanghini and L.Stiaccini for their valuable technical support.
[1] W. Huang, B. Drenkow, and A. Gallimore, in 45th AIAA/ASME/SAE/ASEE Joint Propulsion Conference and Exhibit (2009).

[2] W. Huang, A. Gallimore, and R. Hofer, Journal of Propulsion and Power 27, 553 (2011).

[3] D. Gawron, S. Mazouffre, N. Sadeghi, and A. Hron, Plasma Sources Science and Technology 17 (2008).

[4] G. Bourgeois, S. Mazouffre, and N. Sadeghi, Physics of Plasmas 17 (2010).

[5] W. Hargus Jr. and C. Charles, Journal of Propulsion and Power 24, 127 (2008).

[6] W. Hargus, Jr. and M. Cappelli, Applied Physics B: Lasers and Optics 72, 961 (2001).

[7] R. J. Cedolin, W. A. Hargus, Jr., P. V. Storm, R. K. Hanson, and M. A. Cappelli, Applied Physics B: Lasers and Optics 65, 459 (1997).

[8] W. A. Hargus Jr. and C. S. Charles, Journal of Propulsion and Power 26, 135 (2010).

[9] D. B. Lee, Ph.D. thesis, Graduate School of Engineering and Management, Air Force Institute of Technology, Ohio (2010).

[10] D. Manzella, in NASA report, AIAA-94-3141 (1994).

[11] V. Baranov, Y. Nazarenko, and V. Petrosov, in 27-th International Electric Propulsion Conference, Pasadena, Oct. 14-19 (2001).

[12] B. M. Reid, Ph.D. thesis, University of Michigan, Ann Arbor, MI (2009).

[13] S. Langendorf and M. L. R. Walker, Review of Scientific Instruments 84, 013302 (2013). 